\title{
Serum hepcidin levels in active and inactive Behcet's disease
}

\section{Aktif ve inaktif Behçet hastalığında serum hepsidin düzeyleri}

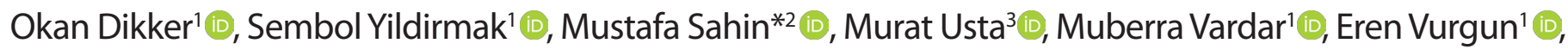 \\ Yuksel Cicek' Mustafa Durmuscan' 10 , Zeynep Altun' 4 , Fehmi Baran 5
}

'Okmeydani Education and Research Hospital, Medical Biochemistry Department, Istanbul/Turkey

${ }^{2}$ Hitit University, Erol Olcok Education and Research Hospital, Medical Biochemistry Department, Corum/Turkey

${ }^{3}$ Giresun University School of Medicine, Medical Biochemistry Department, Giresun/Turkey

${ }^{4}$ Gaziosmanpasa Taksim Education and Research Hospital, Medical Biochemistry Department, Istanbul/Turkey

${ }^{5}$ Okmeydani Education and Research Hospital, Dermatology Department, Istanbul/Turkey

\begin{abstract}
Aim: This study aims to evaluate the alteration of hepcidin synthesis in response to inflammation and to reveal the relationship between hepcidin and acute phase reactants in Behcet's disease (BD) which is also an inflammatory disease.

Material and Methods: That was included 52 BD patients (15 with active, 37 with inactive disease), 13 isolated iron deficiency anemia patients and 13 healthy controls; totally 78 individuals to the study. Patients with chronic disease anemia had 3 active and 1 inactive Behcet's disease. Levels of bioactive hepcidin- 25 were measured by ELISA method.

Result: Low hepcidin levels were found significantly in inactive $B D$ and the main $B D$ groups comparing to healthy controls $(p=0.015, p=0.014$, respectively); in $B D$ with anemia of chronic disease group comparing to healthy controls and isolated iron deficiency anemia group ( $p=0.007, p=0.003$, respectively). Besides it was not found any significant difference between hepcidin levels of active BD group and healthy controls ( $p>0.05)$.

Conclusion: Contrary to its increment pattern in inflammation, increased hepcidin levels in Behcet's patients were not detected. Alternation of serum Interleukin-6 (IL-6) levels with anti-inflammatory treatments affecting hepcidin levels necessitate hepcidin measurement only in Behcet's patients who have been recently diagnosed and/or haven't received a treatment before and evaluation of the measurement together with IL-6 levels.
\end{abstract}

Keywords: Behcet's disease; anemia of chronic disease; hepcidin

Corresponding Author*: Mustafa Sahin, Hitit University, Erol Olcok Education and Research Hospital, Medical Biochemistry Department, Corum/Turkey E-mail: mustafaistanbulx@hotmail.com 


\section{Öz}

Amaç: Bu çalışmada, inflamasyona yanıt olarak hepsidin sentezindeki değişimin değerlendirilmesi ve inflamatuar bir hastalık olan Behçet hastalığında $(\mathrm{BH})$ hepsidin ve akut faz reaktanları arasındaki ilişkiyi ortaya koymak amaçlandı.

Gereç ve Yöntemler: Çalışmaya 52 BH (15 aktif, 37 inaktif hasta) 13 izole demir eksikliği anemisi hastası, 13 sağlıklı kontrol, toplamda 78 katılımcı dâhil edildi. Kronik hastalık anemili hastaların 3'ü aktif, 1 'i inaktif behçet hastasıydı. ELISA yöntemiyle biyoaktif hepsidin-25 düzeylerini ölçüldü.

Bulgular: Inaktif BH ve ana BH gruplarında sağlıklı kontrollere göre anlamlı derecede düşük hepsidin düzeyleri saptandı (sırasıyla $\mathrm{p}=0.015, \mathrm{p}=0.014$ ); hepsidin düzeylerini, kronik hastalık anemisi olan BH grubu ile sağlıklı kontroller ve izole demir eksikliği anemisi grubu karşılaştııılığında ve istatistiksel olarak anlamlı sonuç elde edildi. (sırasıyla $p=0.007, p=0.003$ ). Ayrıca aktif BD grubunun hepcidin düzeyleri ile sağlıklı kontroller arasında anlamlı bir fark bulunamadı ( $p>0.05)$.

Sonuç: İnflamasyondaki artış paterninin aksine, Behçet hastalarında artmış hepsidin seviyelerini tespit edilmedi. Hepsidin seviyelerini etkileyen antiinflamatuar tedavilerin serum İnterlökin-6 (IL-6) düzeylerini değiştirdiğinden, daha önce teşhis edilmiş ve/veya daha önce tedavi almayan Behçet hastalarının IL-6 düzeyleri ile birlikte hepsidin düzeylerinin ölçümü ve değerlendirilmesi gerekmektedir.

Anahtar kelimeler: Behçet hastalığl; kronik hastalık anemisi; hepsidin

\section{Introduction}

Behcet's disease (BD) is an inflammatory vasculitis which is associated with immunological, endothelial and neutrophilic changes [1]. The multi-systemic features of the disease including uveitis with oral and genital ulcerations in particular were first described in 1937 by Hulusi Behcet [2]. Although the complete pathogenesis of the disease which is considered to be an immuno-inflammatory disorder, has not been fully established; genetic factors, infections, immune complexes, antibodies and oxidative stress are the considered contributors [3-5]. A disease-specific laboratory finding linked to BD has not been discovered yet. However, moderate prognosis anemia of chronic disease, elevated sedimentation rate accompanied by increased C-reactive protein (CRP) levels, cryoglobulinemia, leukocytosis and eosinophilia can often be detected [6]. Anemia of chronic disease is the second most common cause of anemia after iron deficiency anemia. It is mostly seen in patients having acute or chronic immune activation $[7,8]$. This type of anemia is also called "anemia of inflammation". Hepcidin whose main function is to inhibit iron releasing from macrophages and enterocytes is known as one of the central regulator hormones of the iron metabolism, but it is also an antimicrobial peptide [9]. Besides "hep", the first part of the name of "hepcidin" comes from liver which is its synthesis place and the second part "cidin" from its in-vitro antimicrobial activity [10]. Its synthesis is regulated in response to anemia, hypoxia and inflammation [11]. Three mature forms of hepcidin exist and the activity of each form is unique [12]. In case of iron overload or inflammation, hepcidin synthesis excessively increases. According to evidence from transgenic mouse models, hepcidin serves to down regulate iron absorption from small intestine, iron transportation from placenta and iron releasing from macrophages. Additionally, hepcidin increases 100 times in anemia of inflammation. It has been asserted that discovery of hepcidin role in iron metabolism may guide to new therapeutic approaches in anemia of inflammation [10].

In this study we aimed to measure the levels of hepcidin whose synthesis is regulated in response to inflammation and to demonstrate the relationship between hepcidin levels and acute phase reactants in patients of Behcet's disease which is also an inflammatory disease.

\section{Material and Methods Design and patients}

We prospectively realized this study on patients applied Dermatology Clinic of Okmeydani Education and Research Hospital. We recorded detailed medical history of all the participants to exclude patients having diabetes mellitus, liver disease, kidney disease, malignancy, infectious disease and any inflammatory disease other than Behcet's disease before. We formed "the main BD group" $(n=52)$ from Behcet's patients 
diagnosed according to International Study Group Diagnostic Criteria [13]. We also selected 26 individuals proved as healthy based on physical examination and laboratory findings for control groups; 13 of them were accepted as completely healthy ("healthy controls") and an another 13 as healthy other than isolated iron deficiency anemia ("isolated iron deficiency anemia group"). Among the main BD group we created one subgroup with active disease consisting of 15 patients ("active BD group") and the other one with inactive disease consisting of 37 patients ("inactive BD group"). We accepted active period of the disease as in addition to oral aphthous ulcers at least one of the following findings: genital ulcers, erythema nodosum-like skin lesions, arthritis and ocular involvement $[14,15]$. Behcet's patients were classified as active BD group who were verifying these findings and as inactive BD group who were not. We also divided the main BD group into three subgroups according to anemia; firstly "non-anemic BD group", secondly "BD with iron deficiency anemia group" and thirdly "BD with anemia of chronic disease group". We defined anemia criteria as hemoglobin level $<13.5 \mathrm{~g} / \mathrm{dL}$ for men and $<12 \mathrm{~g} / \mathrm{dL}$ for women $[16,17]$.We classified anemia with regard to serum ferritin level; $<30 \mathrm{ng} / \mathrm{mL}$ as iron deficiency anemia and >70 $\mathrm{ng} / \mathrm{mL}$ as anemia of chronic disease $[17,18]$.

\section{Collection and storage of biological samples}

We collected venous blood samples at least 8-hour fast for all the tests. We used gel tubes of $10 \mathrm{~mL}$-volume to obtain serum for routine chemistry tests and also for the hepcidin levels. We drew blood samples into $3 \mathrm{ml}$-volume tube containing $\mathrm{K} 3$ EDTA for the hemogram, $3.6 \mathrm{~mL}$-volume tube containing 0.9 $\mathrm{mL}$ of $3.2 \%$ sodium citrate for fibrinogen and $1.6 \mathrm{~mL}$-volume tube containing $0.4 \mathrm{~mL}$ of $3.8 \%$ sodium citrate for erythrocyte sedimentation rate (ESR). Routine chemistry parameters, ferritin, fibrinogen, hemogram, CRP and ESR were analyzed in the same day. Undiluted serum samples were stored at $-80^{\circ} \mathrm{C}$ for hepcidin analyze during 6 months.

\section{Measurements of laboratory tests}

We determined iron and total iron binding capacity (TIBC) levels with photometry by Olympus AU2700 autoanalyzer (Beckman Coulter Inc., CA, USA), ferritin levels with chemiluminescence immunoassay by Beckman Coulter DXI 800 hormone auto-analyzer (Beckman Coulter Inc., CA, USA), fibrinogen levels with optic method by Trinity Biotech MDA II coagulation analyzer, ESR with Westergren method by ERLINE AR auto-analyzer, hemoglobin and red blood cell (RBC) count with flow cytometry by ADVIA 120 auto-analyzer (Siemens Healthcare Diagnostics, IL, USA), CRP levels with nephelometry by BN II auto-analyzer (Siemens Dade Behring, Siemens Healthcare Diagnostics, IL, USA). We calculated transferrin saturation (Trf sat) using (serum iron $\mathrm{x}$ TIBC) $\mathrm{x}$ 100 formula. We measured levels of bioactive hepcidin-25 which is one of the three mature forms of hepcidin (12) by enzyme-linked immunosorbent assay (ELISA) method using DRG International Inc. kit (REF: EIA-4705, New Jersey, USA) in the ELx800 Absorbans Microplate Reader device (BioTek, Winooski, USA). We checked the analytical performance of the method testing two levels of control material within a run. We measured each sample one time.

\section{Performance characteristics of the hepcidin assay}

The analytical measurement range was 0.9 - $140 \mathrm{ng} / \mathrm{mL}$ for hepcidin-25. The minimal detection limit was $0.9 \mathrm{ng} / \mathrm{mL}$. Reported intra-assay and inter-assay CV values were $<5 \%$ and $<12 \%$, respectively. It is claimed for our assay kit that there were no cross reactions between hepcidin-25 and pro-hepcidin.

\section{Ethical committee approval}

Ethical committee approval was taken for this study from the Okmeydani Education and Research Hospital Ethics Committee.

\section{Statistical analyzes}

For statistical analyzes, we used Statistical Package for the Social Sciences 11.5 software package (IBM, SPSS, Chicago, IL, USA).We used Kolmogorov-Smirnov test for normality test of continuous variables. Normal distribution variables were expressed as mean \pm standard deviation and non-normal distribution variables as median and percentiles (25th percentile - 75th percentile). We used one-way ANOVA for between-groups comparison, Tukey HSD or Tamhane's T2 test for post hoc comparison of normal distribution variables. We additionally used Kruskal-Wallis $\mathrm{H}$ test for between-groups comparison, Mann-Whitney $U$ test for post hoc comparison of non-normal distribution variables. We determined correlation between variables using Spearman's rank correlation coefficient (rs) or Pearson product-moment correlation coefficient (r). Trimmed mean which is a statistical measure of central tendency such as mean and median can be used for asymmetric distributions. In this study, for the non-normal distribution variables we used the $5 \%$ trimmed mean which is the mean computed by excluding the $5 \%$ largest and $5 \%$ smallest values from the dataset and having the mean of the remaining $90 \%$. 


\section{Results}

We divided Behcet's patients into two subgroups according to whether they were in active or inactive period of the disease and also into three subgroups according to existence of an anemia (non-anemic BD group, BD with iron deficiency anemia group and BD with anemia of chronic disease group). Then we compared these groups to healthy controls and isolated iron deficiency anemia group. Physical examination of Behcet's patients $(n=52)$ showed that $50 \%$ of the patients had oral aphthous ulcer $(n=26), 11 \%$ of them had genital ulceration $(n=6), 15 \%$ of them had extra-genital ulcerations $(n=8), 36 \%$ of them had arthritis ( $n=19)$ and $25 \%$ of them had ophthalmic findings $(n=13)$. Besides $78 \%$ of the patients $(n=42)$ were using colchicine and/or glucocorticoids. We found significant difference in mean values of hemoglobin $(p<0.0001)$ and fibrinogen $(p<0.008)$ additionally in median values of ferritin $(p<0.0001)$, iron $(p<0.001)$, transferrin saturation $(p<0.001)$, $\operatorname{ESR}(p=0.026), \operatorname{RBC}(p<0.003), \operatorname{CRP}(p=0.008)$ and hepcidin $(p=0.014)$ between healthy controls, isolated iron deficiency anemia and the main BD groups (table 1). We found significant difference in mean values of hemoglobin $(p<0.0001), R B C$ $(p=0.005)$, iron $(p=0.002)$, transferrin saturation $(p=0.008)$ and fibrinogen $(p<0.008)$ additionally between median values of ferritin $(p<0.0001), \operatorname{ESR}(p=0.011), \operatorname{CRP}(p=0.008)$ and hepcidin $(p=0.034)$ between healthy controls, isolated iron deficiency anemia, active BD and inactive BD groups (table 2). We used Mann-Whitney $U$ test for post hoc multiple comparison assuming the significance level of $p<0.016$. We used the $5 \%$ trimmed mean for ferritin, ESR and hepcidin and the $10 \%$ trimmed mean for CRP to calculate the mean difference. According to post-hoc comparison, the median value of hepcidin was significantly low in inactive BD group comparing to healthy controls $(p=0.015$ ) (table 3 ). We found significant difference in median values of hemoglobin $(p<0.0001), \operatorname{RBC}$ $(p=0.005)$, iron $(p=0.005)$, transferrin saturation $(p=0.008)$, TIBC $(p=0.017)$, fibrinogen $(p<0.009), C R P(p=0.002)$ and hepcidin $(p=0.007)$ between healthy controls, isolated iron deficiency anemia, non-anemic BD, BD with iron deficiency anemia and BD with anemia of chronic disease groups (table 4). We used Mann-Whitney $U$ test for post hoc multiple comparison assuming the significance level of $p<0.01$. According to post-hoc comparison, the median value of hepcidin was significantly low in BD with anemia of chronic disease group comparing to isolated iron deficiency anemia group $(p=0.003)$ and healthy controls $(p=0.007)$ (table 5 ). We detected weak negative correlation between hepcidin and fibrinogen ( $r s=-$ 0.332; $\mathrm{p}=0.016$ ) in the main $\mathrm{BD}$ group and strong positive correlation between hepcidin and TIBC ( $r s=0.714 ; p=0.006)$ in isolated iron deficiency anemia group (table 6).

Table 1. Laboratory findings comparison of healthy controls, isolated iron deficiency anemia group and the main BD group

\begin{tabular}{|c|c|c|c|c|}
\hline & Healthy Controls $(n=13)$ & $\begin{array}{l}\text { Isolated Iron Deficiency } \\
\text { Anemia Group }(n=13)\end{array}$ & Main BD Group $(\mathrm{n}=52)$ & $\mathrm{p}$ \\
\hline Ferritin, ng/mL & $49.5(25.7-86.0)$ & $6.4(3.0-24.0)^{f}$ & $23.3(12.0-46.2)^{d}$ & $<0.0001 * *$ \\
\hline Iron, $\mu \mathrm{g} / \mathrm{dL}$ & $86(76-110)$ & $29(20-85)^{c}$ & $51(37-76)^{e}$ & $<0.001 * *$ \\
\hline TIBC, $\mu \mathrm{g} / \mathrm{dL}$ & $352 \pm 31$ & $388 \pm 74$ & $355 \pm 58$ & $0.165^{*}$ \\
\hline Trf sat, $\%$ & $24.5(21.6-33.2)$ & $8.7(4.5-23.7)^{\mathrm{c}}$ & $15.0(10.5-25.2)^{\mathrm{c}}$ & $<0.001 * *$ \\
\hline ESR, mm/h & $10(7-21)$ & $19(13-33)$ & $21(12-34) c$ & $0.026 * *$ \\
\hline $\mathrm{Hgb}, \mathrm{g} / \mathrm{dL}$ & $14.3 \pm 1.3$ & $10.5 \pm 1.2^{\mathrm{f}}$ & $13.0 \pm 1.6^{\mathrm{a}, \mathrm{g}}$ & $<0.0001^{*}$ \\
\hline $\mathrm{RBC}, \mathrm{x} 106 / \mu \mathrm{L}$ & $4.6(4.4-5.1)$ & $4.1(3.8-4.4)^{c}$ & $4.3(4.1-4.7)^{b}$ & $0.003 * *$ \\
\hline Fibrinogen, mg/dL & $249 \pm 35$ & $281 \pm 78$ & $337 \pm 110^{f}$ & $<0.008^{*}$ \\
\hline CRP, mg/L & $3.3(3.2-3.3)$ & $3.3(3.3-3.4)$ & $3.3(3.3-9.5)^{c}$ & $0.008^{* *}$ \\
\hline Hepcidin, ng/mL & $76.8(64.6-110.2)$ & $55.0(38.9-85.6)$ & $41.4(14.7-75.4)^{a}$ & $0.014^{* *}$ \\
\hline
\end{tabular}

CRP: C-reactive protein, ESR: erythrocyte sedimentation rate, RBC: red blood cell, TIBC: total iron binding capacity, Trf sat: transferrin saturation. *p values were calculated using one-way ANOVA analysis.

** $\mathrm{p}$ values were calculated using Kruskal-Wallis $\mathrm{H}$ test.

$\mathrm{aP}<0.05$ (comparing to control group); $\mathrm{b} \mathrm{P}<0.05$ (comparing to isolated iron deficiency anemia group).

$\mathrm{cP}<0.01$ (comparing to control group); $\mathrm{d} \mathrm{P}<0.01$ (comparing to isolated iron deficiency anemia group).

$\mathrm{eP}<0.001$ (comparing to control group); $\mathrm{f}<0.0001$ (comparing to control group); g $\mathrm{P}<0.0001$ (comparing to isolated iron deficiency anemia group). 


\begin{tabular}{|c|c|c|c|c|c|}
\hline & $\begin{array}{l}\text { Healthy Control } \\
(\mathrm{n}=13)\end{array}$ & $\begin{array}{l}\text { Isolated Iron } \\
\text { Deficiency Anemia } \\
\text { Group } \quad(\mathrm{n}=13)\end{array}$ & $\begin{array}{l}\text { Active BD } \\
\text { Group } \\
(\mathrm{n}=15)\end{array}$ & $\begin{array}{c}\text { Inactive BD } \\
\text { Group }(n=37)\end{array}$ & $\mathrm{p}$ \\
\hline Ferritin, ng/mL & $49.5(25.7-86.0)$ & $6.4(3.0-24.0)$ & $44.9(26.5-64.9)$ & $17.0(7.3-35.0)$ & $<0.0001 * *$ \\
\hline Iron, $\mu \mathrm{g} / \mathrm{dL}$ & $92 \pm 29$ & $45 \pm 33$ & $58 \pm 32$ & $59 \pm 29$ & $0.002 *$ \\
\hline TIBC, $\mu \mathrm{g} / \mathrm{dL}$ & $352 \pm 31$ & $388 \pm 74$ & $343 \pm 54$ & $360 \pm 59$ & $0.209^{*}$ \\
\hline Trf sat, \% & $26.0 \pm 7.6$ & $12.8 \pm 10.8$ & $17.4 \pm 11.0$ & $17.1 \pm 9.4$ & $0.008^{*}$ \\
\hline ESR, mm/h & $10(7-21)$ & $19(13-33)$ & $25(20-53)$ & $16(12-32)$ & $0.011 * *$ \\
\hline Hgb, g/dL & $14.3 \pm 1.3$ & $10.5 \pm 1.2$ & $12.9 \pm 1.8$ & $13.0 \pm 1.5$ & $<0.0001^{*}$ \\
\hline $\mathrm{RBC}, \times 106 / \mu \mathrm{L}$ & $4.8 \pm 0.5$ & $4.1 \pm 0.6$ & $4.4 \pm 0.6$ & $4.4 \pm 0.4$ & $0.005^{*}$ \\
\hline Fibrinogen, mg/dL & $249 \pm 35$ & $281 \pm 78$ & $395 \pm 128$ & $314 \pm 94$ & $<0.001 *$ \\
\hline CRP, mg/L & $3.3(3.2-3.3)$ & $3.3(3.3-3.4)$ & $6.1(3.3-14.3)$ & $3.3(3.3-9.2)$ & $0.008 * *$ \\
\hline Hepcidin, ng/mL & $76.8(64.6-110.2)$ & $55.0(38.9-85.6)$ & $39.3(2.9-66.6)$ & $43.4(18.9-79.1)$ & $0.034 * *$ \\
\hline
\end{tabular}

Table 3. Results of Post-hoc multiple comparison analysis realized for ferritin, ESR, CRP and hepcidin parameters after finding the difference statistically significant between healthy controls, isolated iron deficiency, active BD and inactive BD groups according to Kruskal-Wallis $\mathrm{H}$ analysis

\begin{tabular}{|c|c|c|c|c|c|c|}
\hline & & & $\begin{array}{l}\text { Ferritin } \\
(\mathrm{ng} / \mathrm{mL})\end{array}$ & $\begin{array}{c}\mathrm{ESR} \\
(\mathrm{mm} / \mathrm{h})\end{array}$ & $\begin{array}{c}\text { CRP } \\
(\mathrm{mg} / \mathrm{L})\end{array}$ & $\begin{array}{c}\text { Hepcidin } \\
(\mathrm{ng} / \mathrm{mL})\end{array}$ \\
\hline \multirow{6}{*}{ Healthy Controls } & \multirow{2}{*}{$\begin{array}{l}\text { Isolated Iron } \\
\text { Deficiency Group }\end{array}$} & $\begin{array}{l}\text { Trimmed Mean } \\
\text { Difference }\end{array}$ & 44.2 & -8 & -0.7 & 16.3 \\
\hline & & $\mathrm{p}$ & $<0.0001$ & 0.064 & 0.074 & 0.191 \\
\hline & \multirow[t]{2}{*}{ Active BD Group } & $\begin{array}{l}\text { Trimmed Mean } \\
\text { Difference }\end{array}$ & 8.5 & -21 & -3.2 & 32.3 \\
\hline & & $\mathrm{p}$ & 0.662 & 0.003 & 0.002 & 0.034 \\
\hline & \multirow[t]{2}{*}{ Inactive BD Group } & $\begin{array}{l}\text { Trimmed Mean } \\
\text { Difference }\end{array}$ & 28.8 & -9 & -1.7 & 32.7 \\
\hline & & $\mathrm{p}$ & 0.006 & 0.029 & 0.012 & 0.015 \\
\hline \multirow{4}{*}{$\begin{array}{l}\text { Isolated Iron Deficiency } \\
\text { Group }\end{array}$} & \multirow[t]{2}{*}{ Active BD Group } & $\begin{array}{l}\text { Trimmed Mean } \\
\text { Difference }\end{array}$ & -35.7 & -13 & -2.6 & 16.0 \\
\hline & & $\mathrm{p}$ & $<0.0001$ & 0.112 & 0.049 & 0.160 \\
\hline & \multirow[t]{2}{*}{ Inactive BD Group } & $\begin{array}{l}\text { Trimmed Mean } \\
\text { Difference }\end{array}$ & -15.4 & -1 & -1.1 & 16.4 \\
\hline & & $\mathrm{p}$ & 0.022 & 0.868 & 0.397 & 0.084 \\
\hline \multirow[t]{2}{*}{ Active BD Group } & \multirow[t]{2}{*}{ Inactive BD Group } & $\begin{array}{l}\text { Trimmed Mean } \\
\text { Difference }\end{array}$ & 20.3 & 12 & 1.5 & 0.4 \\
\hline & & $\mathrm{p}$ & 0.004 & 0.042 & 0.130 & 0.664 \\
\hline
\end{tabular}




\begin{tabular}{l}
$\begin{array}{l}\text { Table 4. Laboratory findings comparisons of healthy controls, isolated iron deficiency, non-anemic BD, BD with iron } \\
\text { deficiency anemia and BD with anemia of chronic disease groups }\end{array}$ \\
\hline
\end{tabular}

\section{Discussion}

In this study; It was not found significant correlation between hepcidin and ferritin levels in the main $B D$, active $B D$ and isolated iron deficiency groups. Some studies in the literature have reported that hepcidin and ferritin levels are related and positively correlated to each other $[19,20]$ and furthermore some correlation studies on prohepcidin levels have been also realized. Koca et al. [12] reported negative correlation between serum prohepcidin and ferritin levels in healthy control group and that the use of prohepcidin instead of bioactive hepcidin might not be quite reliable. Positive correlation was shown between serum prohepcidin and ferritin levels in chronic renal failure [11]. On the other hand, it has been reported that serum prohepcidin levels are unrelated to ferritin or to other iron parameters [21, 22]. Notwithstanding, our study did not show any correlation between hepcidin and ferritin levels in healthy controls neither in the main BD nor isolated iron deficiency groups. We demonstrated a significant negative correlation between hepcidin and fibrinogen levels in the main BD group, a significant positive correlation between hepcidin and TIBC in isolated iron deficiency group. In active BD group, hepcidin levels were not correlated with any other measured parameters. Hepcidin known as an acute phase protein [19, $23,24]$ was not correlated to none of the other acute phase reactants that we measured, CRP or ferritin. However a negative correlation between hepcidin and fibrinogen was shown. According to these findings we think that a different mechanism affecting hepcidin levels may exist. In this study; we did not find statistically significant difference in hepcidin levels in isolated iron deficiency anemia and active BD groups comparing to healthy controls. Besides we found significantly low hepcidin levels in the main BD and inactive BD groups comparing to healthy controls. We did not find statistically significant difference in hepcidin levels in active BD and inactive $\mathrm{BD}$ groups comparing to isolated iron deficiency anemia.We did not find statistically significant difference in hepcidin levels in isolated iron deficiency anemia, non-anemic 
Volume 11 Number 1 p: 63-72

\begin{tabular}{|c|c|c|c|c|c|c|c|c|c|c|c|}
\hline & & & $\begin{array}{c}\text { Ferritin } \\
\mathrm{ng} / \mathrm{mL}\end{array}$ & $\begin{array}{c}\text { Iron } \\
\mu \mathrm{g} / \mathrm{dL}\end{array}$ & $\begin{array}{l}\text { TIBC } \\
\mu \mathrm{g} / \mathrm{dL}\end{array}$ & $\begin{array}{c}\text { Trf sat } \\
\%\end{array}$ & $\begin{array}{c}\text { Hemoglobin } \\
\mathrm{g} / \mathrm{dL}\end{array}$ & $\begin{array}{l}\mathrm{RBC} \\
\mathrm{x} 1 / \mu \mathrm{L}\end{array}$ & $\begin{array}{c}\text { Fibrinogen } \\
\mathrm{mg} / \mathrm{dL}\end{array}$ & $\begin{array}{l}\mathrm{CRP} \\
\mathrm{mg} / \mathrm{L}\end{array}$ & $\begin{array}{c}\text { Hepcidin } \\
\mathrm{ng} / \mathrm{mL}\end{array}$ \\
\hline \multirow{8}{*}{$\begin{array}{l}\text { Healthy } \\
\text { Controls }\end{array}$} & \multirow{2}{*}{$\begin{array}{l}\text { Isolated Iron } \\
\text { Deficiency } \\
\text { Group }\end{array}$} & $\begin{array}{l}\text { \%5 Trimmed } \\
\text { Mean } \\
\text { Difference }\end{array}$ & 44.137 & 48.37 & -33.04 & 13.950 & 3.671 & 0.689 & -28.01 & -0.795 & 16.236 \\
\hline & & $\mathrm{p}$ & $<0.0001$ & 0.006 & 0.096 & 0.005 & $<0.0001$ & 0.002 & 0.200 & 0.074 & 0.191 \\
\hline & \multirow[t]{2}{*}{$\begin{array}{l}\text { Non-anemic } \\
\text { BD Group }\end{array}$} & $\begin{array}{l}\text { \%5 Trimmed } \\
\text { Mean } \\
\text { Difference }\end{array}$ & 24.484 & 31.39 & -3.48 & 8.954 & 0.678 & 0.162 & -83.99 & -3.641 & 24.462 \\
\hline & & & 0.036 & 0.005 & 0.751 & 0.009 & 0.150 & 0.346 & 0.004 & 0.002 & 0.040 \\
\hline & \multirow{4}{*}{$\begin{array}{l}\text { BD with Iron } \\
\text { Deficiency } \\
\text { Anemia Group } \\
\text { BD with } \\
\text { Anemia of } \\
\text { Chronic } \\
\text { Disease Group }\end{array}$} & $\begin{array}{l}\text { \%5 Trimmed } \\
\text { Mean } \\
\text { Difference }\end{array}$ & 37.338 & 38.19 & -19.70 & 11.365 & 2.488 & 0.616 & -57.69 & -1.140 & 38.443 \\
\hline & & & 0.002 & 0.002 & 0.240 & 0.003 & $<0.0001$ & $<0.001$ & 0.072 & 0.217 & 0.029 \\
\hline & & $\begin{array}{l}\% 5 \text { Trimmed } \\
\text { Mean } \\
\text { Difference }\end{array}$ & -115.292 & 43.24 & 81.78 & 8.483 & 2.545 & 0.760 & -174.34 & -47.854 & 67.067 \\
\hline & & $\mathrm{p}$ & 0.024 & 0.023 & 0.005 & 0.243 & 0.009 & 0.009 & 0.005 & 0.008 & 0.007 \\
\hline \multirow{6}{*}{$\begin{array}{l}\text { Isolated } \\
\text { Iron } \\
\text { Deficiency } \\
\text { Group }\end{array}$} & \multirow[t]{2}{*}{$\begin{array}{l}\text { Non-anemic } \\
\text { BD Group }\end{array}$} & $\begin{array}{l}\% 5 \text { Trimmed } \\
\text { Mean } \\
\text { Difference }\end{array}$ & -19.653 & -16.98 & 29.56 & -4.996 & -2.993 & -0.527 & -55.98 & -2.846 & 8.226 \\
\hline & & $\mathrm{p}$ & 0.002 & 0.075 & 0.251 & 0.042 & $<0.0001$ & $<0.001$ & 0.090 & 0.113 & 0.272 \\
\hline & \multirow[t]{2}{*}{$\begin{array}{l}\text { BD with Iron } \\
\text { Deficiency } \\
\text { Anemia Group }\end{array}$} & $\begin{array}{l}\text { \%5 Trimmed } \\
\text { Mean } \\
\text { Difference }\end{array}$ & -6.799 & -10.18 & 13.34 & -2.585 & -1.183 & -0.073 & -29.68 & -0.345 & 22.207 \\
\hline & & & 0.153 & 0.189 & 0.629 & 0.240 & 0.014 & 0.472 & 0.345 & 0.821 & 0.084 \\
\hline & $\begin{array}{l}\text { BD with } \\
\text { Anemia of } \\
\text { Chronic }\end{array}$ & $\begin{array}{l}\% 5 \text { Trimmed } \\
\text { Mean } \\
\text { Difference }\end{array}$ & -159.429 & -5.13 & 114.82 & -5.467 & -1.126 & 0.071 & -146.33 & -47.059 & 50.831 \\
\hline & Disease Group & $\mathrm{p}$ & 0.003 & 0.955 & 0.005 & 0.213 & 0.192 & 0.607 & 0.023 & 0.020 & 0.003 \\
\hline \multirow{4}{*}{$\begin{array}{l}\text { Non- } \\
\text { anemic } \\
\text { BD Group }\end{array}$} & \multirow{2}{*}{$\begin{array}{l}\text { BD with Iron } \\
\text { Deficiency } \\
\text { Anemia Group }\end{array}$} & $\begin{array}{l}\text { \%5 Trimmed } \\
\text { Mean } \\
\text { Difference }\end{array}$ & 12.854 & 6.80 & -16.22 & 2.411 & 1.810 & 0.454 & 16.30 & 2.501 & 13.981 \\
\hline & & & 0.060 & 0.463 & 0.368 & 0.484 & $<0.0001$ & $<0.001$ & 0.497 & 0.086 & 0.225 \\
\hline & $\begin{array}{l}\text { BD with } \\
\text { Anemia of } \\
\text { Chronic }\end{array}$ & $\begin{array}{l}\% 5 \text { Trimmed } \\
\text { Mean } \\
\text { Difference }\end{array}$ & -139.776 & 11.85 & 85.26 & -0.471 & 1.867 & 0.598 & -100.35 & -44.213 & 42.605 \\
\hline & Disease Group & $\mathrm{p}$ & 0.003 & 0.405 & 0.003 & 0.883 & 0.020 & 0.015 & 0.179 & 0.039 & 0.016 \\
\hline \multirow{2}{*}{$\begin{array}{l}\text { BD with } \\
\text { Iron } \\
\text { Deficiency } \\
\text { Anemia } \\
\text { Group }\end{array}$} & \multirow{2}{*}{$\begin{array}{l}\text { BD with } \\
\text { Anemia of } \\
\text { Chronic } \\
\text { Disease Group }\end{array}$} & $\begin{array}{l}\% 5 \text { Trimmed } \\
\text { Mean } \\
\text { Difference }\end{array}$ & -152.630 & 5.05 & 101.48 & -2.882 & 0.057 & 0.144 & -116.65 & -46.714 & 28.624 \\
\hline & & $\mathrm{p}$ & 0.003 & 0.881 & 0.016 & 0.764 & 0.726 & 0.420 & 0.110 & 0.019 & 0.292 \\
\hline
\end{tabular}

Table 6. Correlations between hepcidin and CRP, fibrinogen, Iron, TIBC, Trf sat and Hemoglobin levels in the main BD, isolated iron deficiency and active BD groups.

\begin{tabular}{|c|c|c|c|c|c|c|c|}
\hline & \multicolumn{2}{|c|}{ Main BD Group $(\mathrm{n}=52)$} & \multicolumn{2}{|c|}{ Isolated Iron Deficiency Group $(\mathrm{n}=13)$} & \multicolumn{2}{|c|}{ Active BD Group $(\mathrm{n}=15)$} \\
\hline & & r or rs & $p$ & $\mathrm{r}$ or rs & $\mathrm{p}$ & r or rs & $p$ \\
\hline \multirow{7}{*}{ Hepcidin } & CRP & $\mathrm{rs}=0.253$ & 0.071 & $\mathrm{rs}=-0.326$ & 0.276 & $\mathrm{rs}=0.135$ & 0.631 \\
\hline & Fibrinogen & $\mathrm{rs}=0.332$ & 0.016 & $\mathrm{rs}=-0.036$ & 0.908 & $\mathrm{rs}=0.108$ & 0.703 \\
\hline & Ferritin & $\mathrm{rs}=0.133$ & 0.348 & $\mathrm{rs}=-0.407$ & 0.168 & $\mathrm{rs}=0.355$ & 0.194 \\
\hline & Iron & $\mathrm{rs}=0.114$ & 0.421 & $\mathrm{rs}=-0.011$ & 0.972 & $\mathrm{rs}=0.280$ & 0.313 \\
\hline & TIBC & $\mathrm{rs}=0.100$ & 0.482 & $\mathrm{rs}=0.714$ & 0.006 & $\mathrm{rs}=-0.032$ & 0.909 \\
\hline & Trf sat. & $\mathrm{rs}=0.082$ & 0.562 & $\mathrm{rs}=-0.225$ & 0.459 & $\mathrm{rs}=0.305$ & 0.270 \\
\hline & Hemoglobin & $\mathrm{rs}=0.186$ & 0.188 & $\mathrm{rs}=0.047$ & 0.879 & $\mathrm{rs}=0.325$ & 0.237 \\
\hline
\end{tabular}


$\mathrm{BD}$ and $\mathrm{BD}$ with iron deficiency anemia groups comparing to healthy controls. Besides we found significantly low hepcidin levels in BD with anemia of chronic disease group comparing to healthy controls. We did not find statistically significant difference in hepcidin levels in non-anemic BD and BD with iron deficiency anemia groups comparing to isolated iron deficiency anemia group. Besides we found significantly low hepcidin levels in BD with anemia of chronic disease group comparing to isolated iron deficiency group. We did not find statistically significant difference in hepcidin levels in BD with iron deficiency anemia and BD with anemia of chronic disease groups comparing to non-anemic BD group. We did not find statistically significant difference in hepcidin levels between $\mathrm{BD}$ with iron deficiency anemia and BD with anemia of chronic disease groups. Contrary to its increment pattern in inflammation and based on the findings stated above, we found significantly low hepcidin levels in BD with anemia of chronic disease, inactive BD and the main BD groups. Due to lack of investigations focusing on hepcidin levels in Behcet's disease we did not have the opportunity to compare our results to the other studies. Nevertheless, we reviewed hepcidin researches which were realized on some other chronic inflammatory diseases: Koca et al. detected higher levels of prohepcidin, the precursor molecule of hepcidin, in rheumatoid arthritis patients comparing to control group and systemic lupus erythematosus patients [12]. They also reported relatively lower prohepcidin levels in the rheumatoid arthritis with anemia of chronic disease subgroup than the rheumatoid arthritis with iron deficiency anemia subgroup. Contrary to these findings related to rheumatoid arthritis, in our Behcet's disease study we did not find significant difference between BD with anemia of chronic disease and BD with iron deficiency anemia groups. Malyszko et al. reported higher hepcidin and ferritin levels in hemodialysis patients than the control group and brought the idea that high levels of hepcidin can be related to functional iron deficiency and the anemia [11]. They found lower serum prohepcidin levels in the anemic subgroup comparing to the non-anemic subgroup of chronic renal failure [11]. It has been asserted that when anemia occurs, hepcidin synthesis decreases in response to anemia and hypoxia [25]. Theurl et al. found higher serum prohepcidin levels in the anemia of chronic disease patient group than the iron deficiency anemia group [26]. They had accepted patients only having history of hemorrhage but not inflammation into their iron deficiency anemia group. Unlikely Theurl et al, we found lower hepcidin levels in BD with anemia of chronic disease group than the isolated iron deficiency group. Our hepcidin levels finding are incoherent to findings of Koca [12], Malyszko [11] and Theurl [26] et al. Contrary to its increment pattern in inflammation and anemia of chronic disease due to up-regulation of its synthesis in response to inflammation [11], we found significantly low hepcidin levels whereas significant high levels of inflammatory markers such as CRP, fibrinogen and ferritin in the main BD group comparing to the control group. We think that the use of immunosuppressive agents as colchicine and glucocorticoids as well as variety of the disease duration and rareness of Behcet's patients with anemia of chronic disease should be under consideration when interpreting these low levels of hepcidin. According to our findings, we think that hepcidin synthesis in BD may be controlled by a different mechanism other than the other inflammatory diseases and that this mechanism may be affected from different molecules. Among these molecules, cytokines are the potential influencers as it was reported that cytokines induce hepcidin synthesis. IL- 6 is the main cytokine mediating hepcidin synthesis $[1,19,23,25]$. IL-6 is also a proinflammatory cytokine having also immunomodulatory functions [27]. It provides synthesis of acute phase reactants and cellular proliferation [28]. Overproduction of IL-6 in some autoimmune diseases and some chronic inflammatory diseases was demonstrated [29]. Evereklioglu et al. [30] showed significantly high serum levels of IL-6 in Behcet's patients comparing to control group and subsequently in active Behcet's patients comparing to inactive Behcet's patients and the control group. They notified that they had excluded patients using immunosuppressive agents including glucocorticosteroids which reduce transcription of proinflammatory cytokines from their study [30]. Hamzaoui et al. [31] reported higher IL-6 levels in their active Behcet's patients group than the control group. Etem et al. [32] compared IL-6 levels between active BD, inactive BD groups and all the patients and they did not find any significant difference among them. They asserted that serum IL-6 levels were depressed due to colchicine which was used for the treatment. Colchicine inhibits the release of fibronectin and alveolar macrophage-derived growth factor from alveolar macrophages. Binding to tubulin, it also inhibits cellular replication and cytokine releasing from polymorphonuclear 
leukocytes [32]. On the other hand, glucocorticosteroids inhibit synthesis of proinflammatory cytokines (IL-1, IL-2, IL-3, IL-6, interferon- $\gamma$, TNF-a) during the inflammation [33]. Kiraz et al. [34] reported that high IL-6 levels tend to decrease after colchicine treatment in family mediterranean fever patients. Sun et al. [35] showed that levomisole and colchicines treatment decreases IL-6, IL-8 and TNF-a levels in Mucocutaneous Behcet disease. Mitigating impact of colchicine on IL-6 levels is clearly indicated in studies of Kiraz S. at al. and Sun A. et al.

In our study, almost all of our patients whether in active or inactive period of the disease were using colchicine and/or a glucocorticosteroid. We think that these anti-inflammatory agents depress serum levels of IL- 6 which is the main cytokine for hepcidin synthesis and that this results on the low serum hepcidin levels in the BD groups. We consider that the measurement of serum levels of hepcidin whose synthesis is under control of inflammation cannot be precisely evaluated in patients using drugs affecting inflammatory process. Further studies should be realized to evaluate serum hepcidin levels together with serum IL-6 levels in Behcet's patients who have been recently diagnosed and/or haven't received a treatment before.

\section{Conclusion}

Contrary to its increment pattern in inflammation, our study exposed significantly low hepcidin levels in BD with anemia of chronic disease, inactive BD and the main BD groups. Alternation of serum IL-6 levels with anti-inflammatory treatments and effect of this alternation on hepcidin levels necessitate hepcidin measurement only in Behcet's patients who have been recently diagnosed and/or haven't received a treatment before and evaluation of the measurement together with IL-6 levels for a clear assessment of hepcidin levels in inflammatory diseases like Behcet's.

\section{Declaration of conflict of interest}

The authors received no financial support for the research and/or authorship of this article. There is no conflict of interest.

\section{References}

1. Erkilic K, Evereklioglu C, Cekmen M, Ozkiris A, Duygulu F, Dogan $\mathrm{H}$. Adenosine deaminase enzyme activity is increased and negatively correlates with catalase, superoxide dismutase and glutathione peroxidase in patients with Behcet's disease: original contributions/clinical and laboratory investigations. Mediators Inflamm 2003; 12: 107-16.
2. Behcet $H$. Uber rezidivivierende aphthose, durchein virus verursachte geschwure am mund, am auge und an den genitalien. Dermatol Wochenschr 1937; 105: 1152-57.

3. Ghate JV, Jorizzo JL. Behcet's disease and complex aphthosis. J Am Acad Dermatol 1999; 40: 1-18.

4. Jorizzo JL, Hudson RD, Schmalstieg FC, Daniels JC, Apisarnthanarax $\mathrm{P}$, Henry JC, et al. Behcet's syndrome: immune regulation, circulating immune complexes, neutrophil migration and colchicine therapy. J Am Acad Dermatol 1984; 10: 205-14.

5. Sandıkcı R, Turkmen S, Guvenen G et al. Lipid peroxidation and antioxidant defence system in patients with active and inactive Behcet's disease. Acta Derm Venereol 2003; 83: 342-46.

6. Champion RH, Burton JL, Burns DA, Breathnach SM, editors. Textbook of dermatogy. 6th Ed. London 1998; 3072-74.

7. Weiss G. Pathogenesis and treatment of anaemia of chronic disease. Blood Rev 2002; 16: 87-96.

8. Means RT Jr. Recent developments in the anemia of chronic disease. Curr Hematol Rep 2003; 2: 116-21.

9. Schranz M, Bakry R, Creus M, Bonn G, Vogel W, Zoller H. Activation and inactivation of the iron hormone hepcidin: Biochemical characterization of prohepcidin cleavage and sequential degradation to N-terminally truncated hepcidin isoforms. Blood Cells Mol Dis 2009; 43: 169-79.

10. Ganz T. Hepcidin, a key regulator of iron metabolism and mediator of anemia of inflammation. Blood 2003; 102: 783-8.

11. Małyszko J, Małyszko JS, Hryszko T, Pawlak K, Mysliwiec M. Is hepcidin a link between anemia, inflammation and liver function in hemodialyzed patients?. Am J Nephrol 2005; 25: 586-90.

12. Koca S, Isik A, Ustundag B, Metin K, Aksoy K. Serum Pro-hepcidin levels in rheumatoid arthritis and systemic lupus erythematosus. Inflammation 2008; 31: 146-53.

13. International study group for Behcet's disease. Criteria for diagnosis Behcet's disease. Lancet 1990; 335: 1078-80.

14. Odabas AR, Karakuzu A, Cetinkaya R, Selcuk Y, Keles S, Bilen H. Increased serum ferritin levels in active Behcet's disease. Int J Clin Pract 2002; 56: 310-11.

15. Bayazit N, Yilmaz M, Oral B et al. Behcet hastalıgında immünolojik aktivasyon belirteçleri. Turk J Dermatol 2008; 2: 34-8.

16. Izaks GJ, Westendorp RG, Knook DL. The definition of anemia in older persons. JAMA 1999; 281: 1714-7.

17. Richer SA. A Practical guide for differentiating between iron deficiency anemia and anemia of chronic disease in children and adults. Nurse Pract 1997; 22: 55 
18. Vreugdenhil G, Baltus CA, Van Eijk HG, Swaak AJ. Anaemia of chronic disease: diagnostic significance of erythrocyte and serological parameters in iron deficient rheumatoid arthritis patients. Br J Rheumatol 1990; 29: 105-10.

19. Nemeth E, Valore EV, Territo M, Schiller G, Lichtenstein A, Ganz T. Hepcidin, a putative mediator of anemia of inflammation, is a type II acute-phase protein. Blood 2003; 101: 2461-63.

20. Dallalio G, Fleury T, Means RT. Serum hepcidin in clinical specimens. Br J Haematol 2003; 122: 996-1000.

21. Taes YE, Wuyts B, Boelaert JR, De VrieseAS, Delanghe JR. Prohepcidin accumulates in renal insufficiency. Clin Chem Lab Med 2004; 42: 387-89.

22. Roe MA, Spinks C, Heath AL, Harvey LJ, Foxall R, Wimperis J, et al. Serum prohepcidin concentration: no association with iron absorption in healthy men; and no relationship with iron status in men carrying HFE mutations, hereditary haemochromatosis patients undergoing phlebotomy treatment or pregnant women. Br J Nutr 2007; 97: 544-49.

23. Nemeth E, Rivera S, Gabayan V, Keller C, Taudorf S, Pedersen BK, et al. IL-6 mediates hypoferremia of inflammation by inducing the synthesis of the iron regulatory hormone hepcidin. J Clin Invest 2004; 113: 1271-76.

24. Lee $\mathrm{P}$, Peng $\mathrm{H}$, Gelbart T, Wang L, Beutler E. Regulation of hepcidin transcription by interleukin-1 and interleukin- 6. Proc Natl Acad Sci USA 2005; 102: 1906-10.

25. Nicolas $G$, Chauvet $C$, Viatte $L$ et al. The gene encoding the iron regulatory peptide hepcidin is regulated by anemia, hypoxia and inflammation. J Clin Invest 2002; 110: 1037-44.

26. Theurl I, Mattle V, Seifert M, Mariani M, Marth C, Weiss G. Dysregulated monocyte iron homeostasis and erythropoietin formation in patients with anemia of chronic disease. Blood 2006; 107: 4142-8.
27. Heinric PC, Castell JV, Andus T. Interleukin- 6 and the acute phase response. Biochem J 1990; 265: 621-36.

28. Kappa A, Piskorski A, Schopf E. Elevated levels of Interleukin 2 receptor in sera of patients with atopic dermatitis and psoriasis. Br J Dermatol 1988; 119: 707-10.

29. Evans CA, Jellis J, Hughes SP, Remick DG, Friedland JS. Tumour necrosis factor-alpha, interleukin-6, and interleukin-8 secretion and theacute-phaseresponse in patients with bacterial and tuberculous osteomyelitis. J Infect Dis 1998; 177: 1582-87.

30. Evereklioglu C, Er H, Turkoz Y, Cekmen M. Serum levels of TNFalpha, sIL-2R, IL-6, and IL-8 are increased and associated with elevated lipid peroxidation in patients with Behçet's disease. Mediators Inflamm 2002; 11: 87-93.

31. Hamzaoui K, Hamzaoui A, Guemira F, Bessioud M, Hamza M, Ayed K. Cytokineprofile in Behcet's disease patients. Scand J Rheumatol. 2002; 31: 205-10.

32. Etem A, Türkmen $S$, Etem $E$ et al. Aktifya da inaktif Behçet hastalığı bulunan hastaların serum interlökin- 6 ve adiponektin seviyeleri. Turkiye Klinikleri J Med Sci 2010; 30: 1837-44.

33. Soni A, Pepper GM, Wyrwinski PM et al. Adrenal insufficiency occurring during septic shock: incidence, outcome, and relationship to peripheral cytokine levels. Am J Med 1995; 98: 266-71.

34. Kiraz S, Ertenli I, Arıcı M et al. Effects of colchicine on inflammatory cytokines and selectins in familial mediterranean fever. Clin Exp Rheumatol 1998; 16: 721-4.

35. Sun A, Wang YP, Chia JS, Liu BY, Chiang CP. Treatment with levamisole and colchicine can result in a significant reduction of IL-6, IL-8 or TNF-alpha level in patients with mucocutaneous type of Behcet's disease. J Oral Pathol Med 2009; 38: 401-5. 